\title{
High-dose-rate intraluminal brachytherapy for paraneoplastic autoimmune multiorgan syndrome
}

\author{
SUN-YOUNG LEE ${ }^{1,2}$, JONG-HYUN KIM ${ }^{2,3}$ and DONG-HYU CHO $\mathrm{CH}^{2,3}$ \\ ${ }^{1}$ Department of Radiation Oncology, Institute for Medical Sciences, Chonbuk National University Medical School; \\ ${ }^{2}$ Institute of Clinical Medicine of Chonbuk National University-Biomedical Research Institute; \\ ${ }^{3}$ Department of Obstetrics and Gynecology, Chonbuk National University Hospital, \\ Jeonju, Jeollabuk-do 561-712, Republic of Korea
}

Received June 17, 2015; Accepted May 26, 2016

DOI: $10.3892 /$ etm.2016.3458

\begin{abstract}
Paraneoplastic autoimmune multiorgan syndrome (PAMS), also known as paraneoplasic pemphigus, involves the skin, internal organs and mucosa. PAMS-associated mortality may occur as a result of autoantibody formation against internal tumors and their infiltration into organs other than the skin lesions that characterize PAMS. The most common symptoms of PAMS include pain associated with continuous oral ulceration and resistance to pharmacological treatment. The present study reports the case of a 42-year-old female patient who was admitted with an 8-month history of erosive skin lesions within the trunk region, oral mucosa and vaginal mucosa. The patient was diagnosed with PAMS based on computed tomography scans and histological analyses of the lesions. The lymphoid hyperplasia in the retroperitoneum and lesions in the vaginal mucosa and trunk area were improved following pharmacological treatment and resection of the lymph node showing hyperplasia. However, the oral lesion was treated with intraluminal brachytherapy due to its resistance to long-term pharmacological treatment. The majority of the lesions were improved following treatment, in the absence of any severe side effects. In addition, neither worsening nor progression of the oral lesion was observed during the 4-year follow-up period.
\end{abstract}

\section{Introduction}

Paraneoplastic pemphigus, which was initially described by Anhalt et al (1), is characterized by non-specific pemphigus accompanying mucosal skin lesions showing erosion and

Correspondence to: Professor Jong-Hyun Kim, Department of Obstetrics and Gynecology, Chonbuk National University Hospital, 634-18 Geumam-dong, Jeonju, Jeollabuk-do 561-712, Republic of Korea

E-mail: hyeon69@jbnu.ac.kr

Key words: paraneoplastic autoimmune multiorgan syndrome, oral lesion, intraluminal brachytherapy blisters, which occur as a result of the production of autoantibodies against internal tumors. Skin lesions associated with autoantibodies may not only be pemphigus lesions, but also erythema multiforme-like lesions, graft-versus-host disease-like lesions and lichen planus lesions $(1,2)$. These diseases may also cause mortality due to the infiltration of autoantibodies into other organs, thus leading to the term paraneoplastic autoimmune multiorgan syndrome (PAMS) (2).

Lymphoid hyperplasia is the rapid growth and proliferation of normal cells that resemble lymph tissue, and the majority of lymphoid hyperplasia cells are T lymphocytes $(2,3)$. Neoplasms associated with lymphoid hyperplasia include Castleman's disease, non-Hodgkin's lymphoma and thymoma, and these tumors have been frequently associated with PAMS (2). Conversely, sarcoma and malignant melanoma have rarely been associated with PAMS (3). The most common symptoms of PAMS are persistent oral lesions that are typically resistant to pharmacological treatment (4-7). Brachytherapy can be attempted when considering the accessibility of the disease lesion, to minimize the side effects to the adjacent organ and to increase the therapeutic radiation dose (8).

The present study is, to the best of our knowledge, the first to report the results of intraluminal brachytherapy application for the treatment of a persistent and pharmacological-resistant oral erosive ulcer in a 42-year-old female patient with giant lymph node hyperplasia (Castleman's disease) and erosive skin lesions in the vaginal mucosa and trunk region.

\section{Case report}

A 42-year-old female patient visited Chonbuk National University Hospital (Jeonju, Republic of Korea) with an 8-month history of erosive skin lesions occurring in the trunk region, oral mucosa and vaginal mucosa. The patient's medical history and family history were normal. An abdominal computed tomography (CT) scan (date, 18/6/2008) was performed following the detection of elevated CA125 (86.4 U/ml; normal, 0-37 U/ml) and CA19-9 (185.46 U/ml; normal, $<37 \mathrm{U} / \mathrm{ml}$ ) levels in a blood examination. A retroperitoneal mass identified in the abdominal CT scan was removed (date, 25/6/2008) and the patient was diagnosed with 


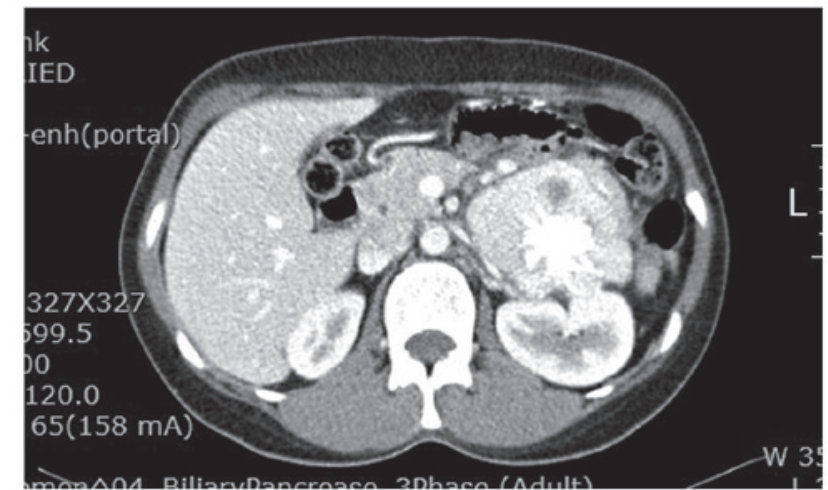

Figure 1. Axial abdominal computed tomography scan. A lobulated hypovascular mass of $12 \mathrm{~cm}$ was observed between the stomach and left kidney of the patient, which was accompanied by central dense calcification.

Castleman's disease according to this biopsy (date, 30/6/2008) (Fig. 1). The biopsy was a large $(12 \times 6.5 \times 6.8 \mathrm{~cm})$ mass with multicentric, hyaline vasular lymph node hyperplasia. The study was approved by the Institutional Review Board of Chonbuk National University Hospital and was conducted according to the Declaration of Helsinki regarding biomedical research involving human subjects. A detailed explanation of the study was provided to the patient, and written informed consent was obtained from the patient.

Biopsies of the skin lesions were performed, and hematoxylin and eosin-stained pathology sections were classified according to their histological features, analyzed under a microscope (BX50; Olympus Corporation, Tokyo, Japan) . The trunk lesions showed non-caseating granulomous features and positive lichen planus under low magnification, and chronic inflammatory cell infiltration, numerous blood vessels, lymphohistiocytic granuloma infiltrate and multicentric giant cells under high magnification (Fig. 2A). In the skin lesions from the vaginal mucosa, epidermal labia majora-acanthosis, separation of the dermoepidermal junction, destruction of the basal layer and deposition of lymphoid cells and neutrophils in the dermis were observed under low magnification, and dyskeratosis, exocytosis, acantholysis and squamous metaplasia of the epidermis and keratinocytes were observed under high magnification (Fig. 2B). The tongue was ulcerated and lacked the epithelium, and dense lymphoid cell infiltration into the tongue was observed under low magnification. In addition, acanthosis and dense lymphoid cell infiltration were observed under high magnification. Immunofluorescence (date, 7/8/2008) revealed fibrinogen deposition along the basement membrane (Fig. 2C). For the immunofluorescence assay, the tissue specimen was incubated for $1 \mathrm{~h}$ at room temperature using a human IgG fluorescence-labeled antibody (cat. no. 23310; Pierce Biotechnology; Thermo Fisher Scientific, Inc., Waltham, MA, USA; dilution, 1:200).

The patient was diagnosed with PAMS (7/8/2008) following analyses of the tissue obtained from the retroperitoneal mass and skin lesions. Following the diagnosis, retroperitoneal mass resection was performed to alleviate pain caused by the mass and pharmacological treatment was performed for skin and mucosal lesions. Pharmacological treatment consisted of a 4-month treatment regime of twice
A

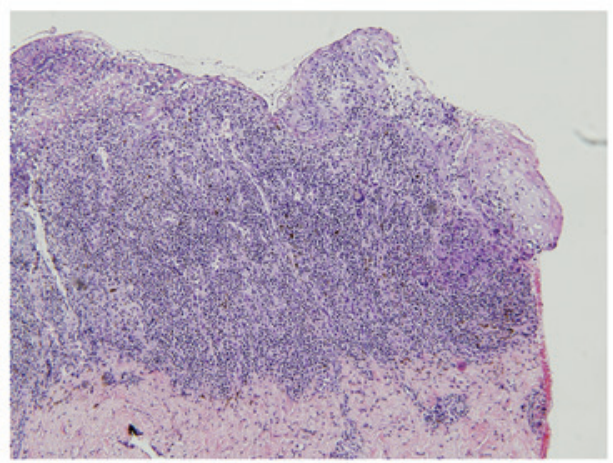

B

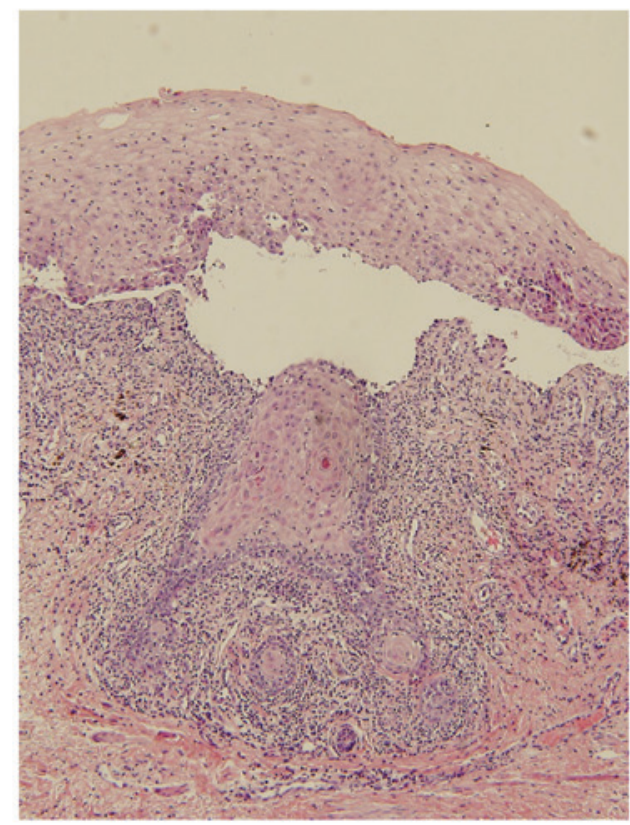

C

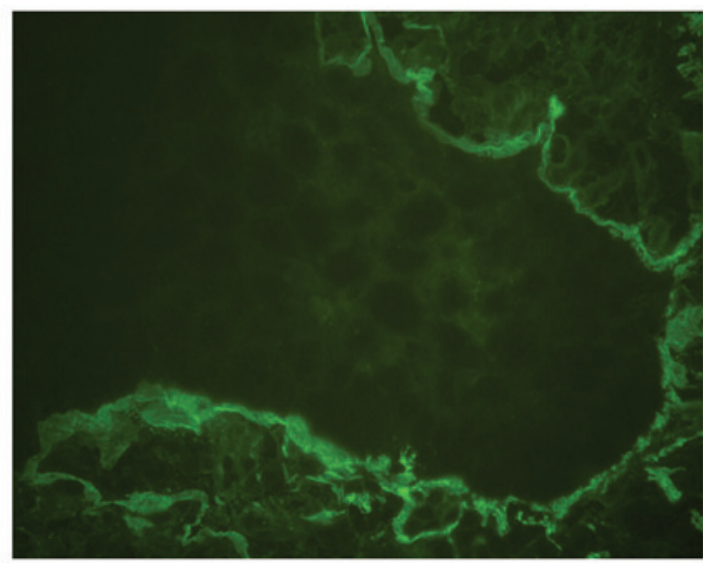

Figure 2. Biopsies of the trunk, vaginal mucosa and oral mucosa skin lesions (A) In the trunk skin lesions, chronic inflammatory cell infiltration, numerous blood vessels, lymphohistiocytic granuloma infiltrate and multicentric giant cells were observed under high magnification [hematoxylin \& eosin (H\&E); magnification, x100]. (B) In the vaginal mucosa lesions, dyskeratosis, exocytosis, acantholysis and squamous metaplasia of the epidermis and keratinocytes were observed under high magnification (H\&E; magnification, x100). (C) Immunofluorescence revealed fibrinogen deposition along the basement membrane of the oral mucosa lesion.

daily methotrexate (50 mg; JW Pharmaceutical, Seoul, Republic of Korea) and thalidomide (200 mg; TayTech BioGen, Seoul, Republic of Korea) administration, followed by a one-time administration of azaprine $(100 \mathrm{mg}$; Korean 


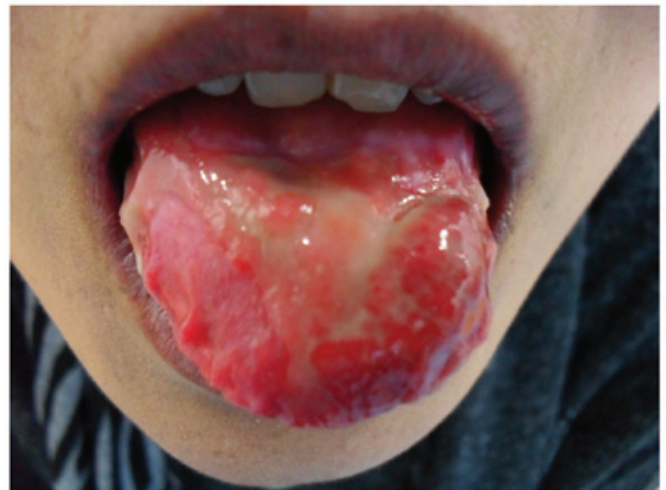

Figure 3. Oral lesion prior to brachytherapy. The oral lesion was limited to the anterior half of the tongue. No ulcerous lesions were observed on the buccal mucosa.

A

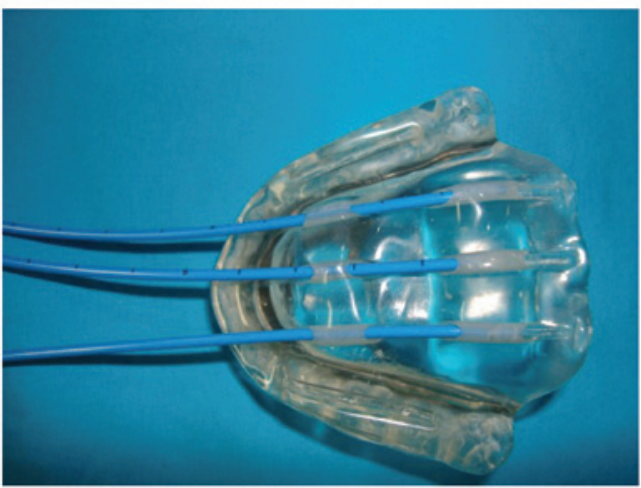

B

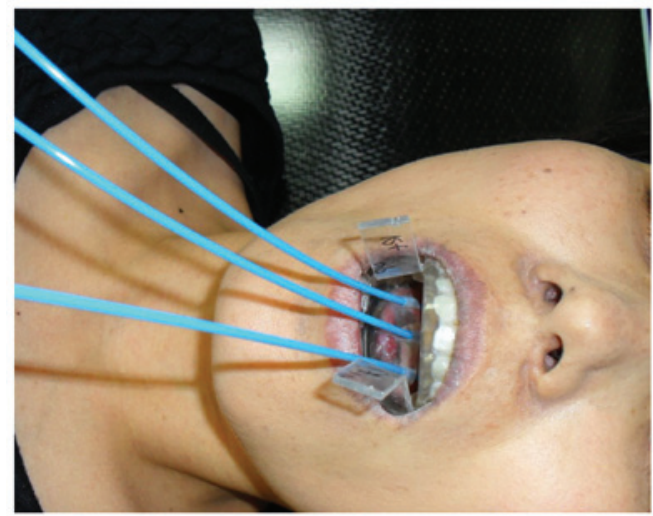

C

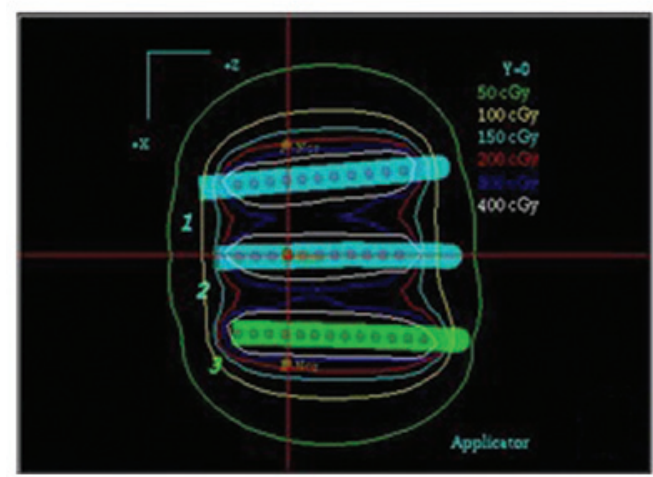

Figure 4. Brachytherapy for the treatment of the oral lesion. (A) The mold for the lower jaw consisted of three $6 \mathrm{~F}$ catheter holes with $1-\mathrm{cm}$ intervals to permit the insertion of the catheter for brachytherapy. (B) The mold for the lower jaw was designed to have a $2-\mathrm{mm}$ lead plate to minimize radiation exposure to the gingiva and teeth, which were located adjacent to the tongue lesion. (C) Treatment was performed by inserting a Lumencath ${ }^{\circledR}$ catheter $(6 \mathrm{~F}$ $150 \mathrm{~cm}$ ) for brachytherapy.

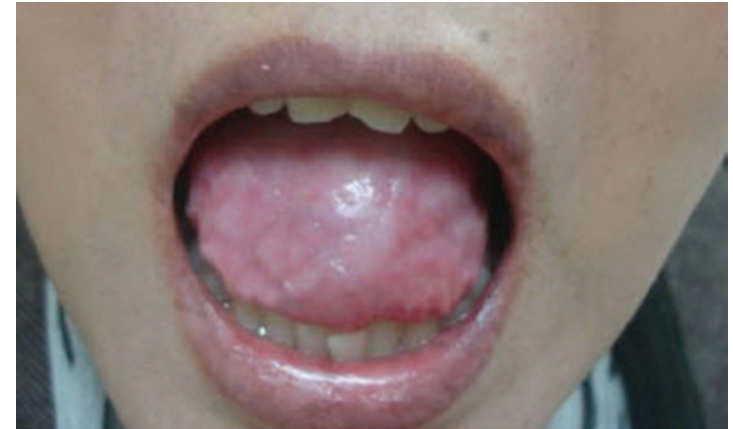

Figure 5. Oral lesion following brachytherapy. The majority of the lesions on the tongue had disappeared by the end of the brachytherapy, and aggravation of the disease was not observed during the 4-year follow-up period.

United Pharmaceutical, Inc., Seoul, Republic of Korea), an immunosuppressive drug, and $10 \mathrm{mg} /$ day prednisolone (Korea Pharma, Seoul, Republic of Korea) for 1 year. The majority of the skin lesions in the trunk and vaginal mucosa were successfully treated, albeit the erosive lesion in the tongue was non-responsive to systemic pharmacological treatment and caused persistent pain. Therefore, $\mathrm{AgNO}_{3}$ (Gana Chem Co., Ltd., Ulsan, Republic of Korea) treatment and local injection of Tamceton (40 mg; HanAll BioPharma Co., Ltd., Seoul, Republic of Korea) were administered 20 and 15 times, respectively. However, the tongue lesion was non-responsive to these treatments, and the patient underwent intraluminal brachytherapy.

Oral lesions were limited to the anterior half of the tongue, and no ulcerous lesions were observed in the buccal mucosa or gingiva (Fig. 3). Intraluminal brachytherapy was performed (date, 7/1/2010) to treat locally-located lesions, prevent damage to the gingiva and enhance the radiation dose effect. The device for oral brachytherapy was designed using acrylic resin and consisted of separate molds for the upper and lower jaws. The mold for the lower jaw consisted of three $6 \mathrm{~F}$ catheter holes with $1-\mathrm{cm}$ intervals to insert the catheter for brachytherapy (Fig. 4A). The mold for the lower jaw constituted 2-mm lead plates to minimize the radiation exposure to the gingiva and teeth during the therapy, which were located adjacent to the tongue lesion. A 2-mm lead plate was also inserted into the mold for the upper jaw during each therapy to reduce the radiation exposure to the palate (Fig. 4B). The radiation dose for each therapy was limited to $\leq 15 \mathrm{cGy}$. Treatment was performed using a Lumencath ${ }^{\circledR}$ catheter $(6 \mathrm{~F} 150 \mathrm{~cm}$; Elekta Instrument AB Stockholm, Stockholm, Sweden) for brachytherapy. Radiation treatment consisted of 2 Gy per session and two sessions per week, using an Iridium-192 high-dose-rate brachytherapy system (microSelectron HDR afterloader; Elekta Instrument AB Stockholm) (Fig. 4C). The patient was exposed to a total of 40 Gy radiation during 20 therapy sessions.

Grade 2 radiation-induced oral mucositis (9) was observed during the treatment. Chronic side effects were not observed during the follow-up period and the majority of the tongue lesions were successfully treated following brachytherapy (Fig. 5). No disease progression was observed during the 4-year follow-up period; the patient's oral disease lesions completely healed. 


\section{Discussion}

Pemphigus vulgaris is characterized by acantholysis and intraepidermal blister formation, which are caused by the loss of normal interactions between cells due to the production of autoantibodies against the surface proteins of keratinocytes (10). However, Anhalt et al (1) examined a case of pemphigus vulgaris showing non-specific clinical symptoms, including painful erosive mucosal lesions and various skin rashes, that were associated with the production of autoantibodies against a tumor, which they termed paraneoplastic pemphigus. Subsequently, Nguyen et al (11) suggested PAMS as an alternative and more appropriate name for paraneoplastic pemphigus for various reasons. First, the heterogeneity of clinical characteristics can be expressed as clear rash without blisters against the cross-section of the cells; second, autoimmune multiorgan syndrome targeting epidermal cells and internal organs is commonly observed in patients with paraneoplastic pemphigus; and third, respiratory failure as a result of changes in bronchial epithelial cells is observed, despite not typically being associated with pemphigus.

Anhalt et al (1) suggested that paraneoplastic pemphigus be diagnosed using the results of direct and indirect immunofluorescence analyses, immunoprecipitation assays, an analysis of clinical symptoms, including mucosal lesions with pain, various systemic skin lesions and internal organ tumors, and characteristic histological findings, including necrosis of keratinocytes, disjunction of intraepidermal pickle cells, vacuolization of the basal cell layer and dermatitis in the cell junction area. However, Camisa and Helm (6) reported that PAMS could not be diagnosed in some cases due to insufficient findings upon examination or a condition that prohibited such examinations. Therefore, authors of that study proposed novel criteria for the diagnosis of PAMS based on distinguishing between major and minor findings (6). The major findings included various types of skin rashes, associated internal tumors and characteristic immunoprecipitation results, whereas the minor findings included the confirmed disjunction of pickle cells via histological analysis, precipitation of immunoreactants between the basal cell layer and cells upon direct immunofluorescence and positive findings in indirect immunofluorescence analyses using epithelial cells from rat urinary bladders (6). Using this method, PAMS may be diagnosed if all the three major findings are identified or if any two of the major findings and any two of the minor findings are observed (6). In the present study, the patient was diagnosed with PAMS since she showed two of the major findings and two of the minor findings identified previously $(1,4,10)$.

PAMS has been rarely reported and its accurate prevalence is not fully known. This may be because many cases of PAMS are misdiagnosed as other diseases, including erythema multiforme, Stevens-Johnson syndrome, toxic epidermal necrolysis, lichen planus, pemphigus and graft-versus-host disease, due to its diversity of clinical manifestations and histopathological findings (11).

At present, PAMS is considered an autoimmune disease and thus is treated with palliative treatments, including removal of the symptom-causing mass or administration of immunosuppressive drugs or adrenal cortical hormones (3-7).
However, a clear method for its treatment has yet to be established (3-8).

Painful oral erosive ulcers and various forms of skin rashes are typical skin lesions associated with PAMS (2). In particular, stomatitis is a common clinical symptom in PAMS patients (1,9-11). Stomatitis is observed in the earliest stage of the disease and shows the strongest resistance to treatment, such that it is often the most persistent of the associated symptoms. It typically occurs on the lateral side of the tongue and extends towards the lip versmilion, thus causing pain. Notably, PAMS has been shown to have more severe symptoms and a wider range of clinical manifestations, as compared with normal pemphigus $(10,11)$.

In the present case, the first symptom experienced by the patient was an erosive lesion in the oral mucosa and tongue, which was associated with severe pain. Other symptoms experienced by the patient, including skin lesions of the trunk and vaginal mucosa, were improved following surgical resection of the mass from the retroperitoneum, which was associated with the patient's Castleman's disease, as well as treatment with an adrenal cortical hormone, methotrexate, thalidomide and an immunosuppressive drug, azaprine. However, the oral lesion was unresponsive to the pharmacological treatment and surgical resection, and was the cause of persistent severe pain.

Previous studies have reported that autoimmune disorders may be sensitive to radiation $(12,13)$. Therefore, radiation therapy was considered for the present case. In the present study, the patient underwent brachytherapy to minimize radiation exposure to the surrounding organs. As a result of brachytherapy, the lesions in the tongue were controlled without causing serious acute or chronic side effects. In addition, neither worsening nor progression of the lesion was observed during the 4-year follow-up period.

In conclusion, the present study has demonstrated that brachytherapy is a promising therapeutic strategy for the persistent and drug-resistant oral lesions that are often observed in patients with PAMS. However, regular observation of the patient may be required due to the slow response of PAMS to treatment and the high rate of relapse of this disease.

\section{Acknowledgements}

The present study was supported by funds from the Institute of Clinical Medicine of Chonbuk National University, Biomedical Research Institute, Chonbuk National University Hospital.

\section{References}

1. Anhalt GJ, Kim SC, Stanley JR, Korman NJ, Jabs DA, Kory M, Izumi H, Ratrie H III, Mutasim D and Ariss-Abdo L: Paraneoplastic pemphigus. An autoimmune mucocutaneous disease associated with neoplasia. N Engl J Med 323: 1729-1735, 1990.

2. Bystryn JC, Hodak E, Gao SQ, Chuba JV and Amorosi EL: A paraneoplastic mixed bullous skin disease associated with anti-skin antibodies and a B-cell lymphoma. Arch Dermatol 129: 870-875, 1993.

3. Sehgal VN and Srivastava G: Paraneoplastic pemphigus/paraneoplastic autoimmune multiorgan syndrome. Int J Dermatol 48: 162-169, 2009.

4. Anhalt GJ and Nousari C: Paraneoplastic Pemphigus. In: Fitzpatrick's Dermatology in General Medicine. 7th edition. Wolff K, Goldsmith LA, Katz SI, Gilchrest BA, Paller AS and Leffell DJ (eds). McGraw-Hill Education, New York, NY, pp468-474, 2008. 
5. Lane JE, Woody C, Davis LS, Guill MF and Jerath RS: Paraneoplastic autoimmune multiorgan syndrome (paraneoplastic pemphigus) in a child: Case report and review of the literature. Pediatrics 114: e513-e516, 2004.

6. Camisa $\mathrm{C}$ and Helm TN: Paraneoplastic pemphigus is a distinct neoplasia-induced autoimmune disease. Arch Dermatol 129: 883-886, 1993.

7. Wade MS and Black MM: Paraneoplastic pemphigus: A brief update. Australas J Dermatol 46: 1-8, 2005

8. Huang CJ, Hou MF, Luo KH, Wei SY, Huang MY, Su SJ, Kuo HY, Yuan SS, Chen GS, Hu SC and Chuang HY: RTOG, CTCAE and WHO criteria for acute radiation dermatitis correlate with cutaneous blood flow measurements. Breast 24: 230-236, 2015.

9. Garran C, Montesdeoca N and Martinez MR: Treatment of upper gum cercinima with high-dose-rate customized-mold brachytherapy. Brachytherapy 7: 267-269, 2008.

10. Heymann WR: Paraneoplastic autoimmune multiorgan syndrome. J Am Acad Dermatol 51: 631-632, 2004.
11. Nguyen VT, Ndoye A, Bassler KD, Shultz LD, Shields MC, Ruben BS, Webber RJ, Pittelkow MR, Lynch PJ and Grando SA: Classification, clinical manifestations and immunopathological mechanisms of the epithelial variant of paraneoplastic autoimmune multiorgan syndrome: A reappraisal of paraneoplastic pemphigus. Arch Dermatol 137: 193-206, 2001.

12. Billet SE, Grando SA and Pittelkow MR: Paraneoplastic autoimmune multiorgan syndrome: Review of the literature and support for a cytotoxic role in pathogenesis. Autoimmunity 39: 617-630, 2006.

13. Neuhof D and Debus J: Outcome and late complications of radiotherapy in patients with unicentric Castleman disease. Acta Oncol 45: 1126-1131, 2006. 